\title{
NGHIÊN CỨU CÔNG THỨC TÍNH CHUYỂN Độ CAO CHUẨN TỪ' Hệ TRIỀU TRUNG BìNH VỀ Hệ TRIỀU 0
}

\author{
PGS. TSKH. HÀ MINH HÒA \\ KS. NGUYẾN TH! THANH HƯƠNG \\ Viện Khoa học Đo đạc và Bản đồ
}

\section{Tóm tắt:}

Bài báo khoa học này đã thực hiện việc mô tả chính xác các giá trị thế năng thực của Mặt Geoid và mặt vật lý Quả đất trong các hệ triều khác nhau và chứng minh công thức chuyển độ cao chuẩn từ hệ triều trung bình về hệ triều 0 . Các kết quả nghiên cứu trong bài báo này đã tạo cơ sở cho việc giải quyết bài toán hoàn thiện hệ độ cao quốc gia gắn liền với việc xây dựng Khung quy chiếu không gian quốc gia trong tương lai.

\section{1. Đặt vấn đề}

2 iệc giải quyết hàng loạt các bài toán hiện đại của Trắc địa cao cấp hiện nay như hoàn thiện hệ độ cao gắn liền với việc xây dựng khung quy chiếu không gian, xây dựng mô hình Quasigeoid độ chính xác cao đều gắn liền với việc sử dụng các đại lượng như độ cao chuẩn độ chính xác cao được xác định từ kết quả đo thủy chuẩn độ chính xác cao, độ cao trắc địa độ chính xác cao được xác định từ kết quả đo đạc GNSS và xử lý trong Khung quy chiếu Quả đất ITRF (International Terrestrial Reference Frame) và độ cao Geoid được xác định từ mô hình trọng trường Quả đất EGM (Earth Gravitational Model). Tuy nhiên các đại lượng nêu trên lại được xác định trong các hệ triều khác nhau. Dưới sức hút của Mặt trăng và Mặt trời xẩy ra hiện tượng triều trên bề mặt Quả đất mà dưới tác động của hiện tượng này, cả mặt Geoid lẫn mặt vật lý của Quả đất luôn bị biến thiên.

Sóng trên các đại dương là kết quả tổng hợp các loại sóng được sinh ra bởi sức hút của Mặt trăng và Mặt trời. Theo kết quả đo đạc trọng lực độ chính xác cao đã xác định được rằng [4], sự dịch chuyển đứng của bề mặt Quả đất dưới sức hút của Mặt trăng và Mặt trời có thể đạt tới $40-50 \mathrm{~cm}$ trên lục địa và $19 \mathrm{~m}$ trên các đại dương. Sự biến dạng triều của Quả đất do sức hút của Mặt trăng và Mặt trời có thể làm xê dịch vị trí mặt bằng và độ cao ở mức $40 \mathrm{~cm}$ trong vòng 6 giờ [6].

Các hiệu ứng trực tiếp và gián tiếp đều có 2 dạng; chu kỳ (periodic, cyclic) và thường trực (permanent). Hiệu ứng thường trực là hiệu ứng không bị loại bỏ nhờ việc lấy giá trị trung bình các kết quả đo đạc cùng một đại lượng, hiệu ứng chu kỳ là hiệu ứng có thể loại bỏ nhờ lấy giá trị trung bình các kết quả đo đạc cùng một đại lượng. Hiệu ứng triều trực tiếp được gây ra do sức hút của Mặt trăng và Mặt trời. Hiệu ứng triều gián tiếp được gây ra do sự phần bố lại vật chất trong lòng Quả đất diễn ra theo các quy luật tự nhiên dưới tác động của hiện tượng triều khi tính đến sự đàn hồi của Quả đất. Việc tính đến hiệu ứng triều gián tiếp được thực hiện nhờ các số Love. Do đó thực tế các công tác đo đạc và tính toán trắc địa đều liên quan đển mặt Geoid và mặt vật lý của Quả đất bị ảnh hưởng của hiệu ứng triều gián tiếp, tức chúng ta làm việc với các đại lượng thuộc hệ triều 0. 
Có 3 dạng hệ thống triều [9]:

- Hệ thống triều trung bình (Mean Tide System): Các hiệu ứng triều chu kỳ đều bị loại bỏ, nhưng biến dạng triều thường trực (cả trực tiếp và gián tiếp) vẫn còn được lưu lại.

- Hệ thống triều Zero (0) (Zero Tide System): Hiệu ứng triều trực tiếp bị loại bỏ, nhưng hiệu ứng triều gián tiếp vẫn còn tồn tại.

- Hệ thống không phụ thuộc triều (Free Tide or Non - Tide System): Tất cả các hiệu ứng triều (trực tiếp và gián tiếp) đều được loại bỏ.

Do hiệu ứng triều gián tiếp không thể bị loại bỏ trong các kết quả đo đạc, nên để thống nhất sử dụng các đại lượng khác nhau trong quá trình giải quyết các bài toán hiện đại của Trắc địa cao cấp, tại cuộc họp 1983 IAG/UGG của Hội Trắc địa quốc tế (International Association of Geodesy - IAG) năm 1983 tại Hamburg đã ra Nghị quyết 16 [7], theo đó các hệ thống được sử dụng trong Trắc địa phải tương ứng với Hệ thống triều 0 (Zero). Điều này có nghĩa là các nhà trắc địa sẽ sử dụng hệ thống triều với hiệu ứng triều trực tiếp bị loại bỏ, nhưng hiệu ứng triều gián tiếp vẫn còn tồn tại.

Khi đo đạc thủy chuẩn hạng I, II trên bề mặt vật lý của Quả đất chúng ta tiến hành đo đi và đo về. Do đó kết quả xác định chênh cao trung bình đã loại bỏ ảnh hưởng của các sóng triều biến đổi theo chu kỳ. Tuy nhiên ảnh hưởng của sóng vùng là tác nhân gây ra hiệu ứng triều trực tiếp thường trực vẫn chưa bị loại bỏ. Do đó các chênh cao trung bình trong mang lưới thủy chuẩn quốc gia và các độ cao chuẩn của các mốc thủy chuẩn hạng $\mathrm{I}$ và Il đều thuộc hệ triều trung bình.

Lý thuyết về việc hiệu chỉnh các chênh cao đo và độ cao chuẩn từ hệ triều trung bình về hệ triều 0 ít được đề cập trong các tài liệu của Trắc địa cao cấp.

Trong quá trình xây dựng Hệ độ cao Châu Âu EVRF 2007 (European Vertical Reference Frame), thực hiện Nghị quyết 16 của Hội Trắc địa quốc tế năm 1983, các nhà trắc địa Châu Âu đã định nghĩa EVRF 2007 là Hệ độ cao thuộc hệ triều 0 [11]. Khi bình sai mạng lưới Châu Âu, thay cho độ cao chuẩn $H_{M}^{\gamma}$ của điểm $M$ trên mặt vật lý của Quả đất làm ẩn số, các nhà khoa học Châu Âu sử dụng đại lượng địa thế năng $\mathrm{C}_{\mathrm{M}}$ liên hệ với độ cao chuẩn $H_{M}^{\gamma}$ theo công thức

$$
H_{M}^{\gamma}=\frac{W_{0}-W_{M}}{\bar{\gamma}}=\frac{C_{M}}{\bar{\gamma}},
$$

ở đây $\mathrm{W}_{\mathrm{M}}$ - thế năng trọng trường thực tại điểm $\mathrm{M}, \mathrm{W}_{0}$ - thế năng trọng trường thực trên mặt Geoid làm khởi tính cho hệ độ cao, $\bar{\gamma}$ - giá trị trung bình của lực trọng chuẩn tại điểm $M$ được xác định theo công thức

$$
\bar{\gamma}=\gamma_{0}-\frac{0,3086 \cdot H_{M}^{\gamma}}{2}+\frac{0,072 \cdot 10^{-6}\left(H_{M}^{\gamma}\right)^{2}}{2}<m G a l>
$$

còn $\gamma_{0}$ - gia tốc lực trọng trường chuẩn trên mặt Ellipsoid được sử dụng.

Để chuyển đại lượng địa thế năng $\left(\mathrm{C}_{\mathrm{M}}\right)_{\mathrm{m}}$ từ hệ triều trung bình (Mean Tide System) về giá trị $\left(C_{M}\right)_{Z}$ trong hệ triều 0 (Zero Tide System), trong tài liệu [11] đã đưa ra công thức:

$$
\left(C_{M}\right)_{Z}=\left(C_{M}\right)_{m}+W_{V}
$$


ở đây $W_{V}$ - thế triều của sóng vùng được xác định theo công thức [10]:

$$
W_{V}=97220-288410 \cdot \operatorname{Sin}^{2} B-1950 \cdot \operatorname{Sin}^{4} B \quad<\mathrm{m} \cdot \mathrm{mGal}>
$$

ở đây $B$ - vĩ độ trắc địa của điểm $M$ trên mặt vật lý của Quả đất.

Nếu ký hiệu

$$
\Delta \rho_{0}=\frac{W_{V}}{\bar{\gamma}}
$$

là độ dịch chuyển đứng của mặt Geoid do tác động của sóng vùng, thêm vào đó theo tài liệu [4], đại lượng $\Delta \rho_{0}>0$ (tức mặt Geoid được nâng lên) trong khu vực được giới hạn bởi vĩ tuyến với vĩ độ $|\varphi|<35^{\circ} 16^{\prime}$ trong đó có Việt Nam, và đại lượng $\Delta \rho_{0}<0$ (tức mặt Geoid được hạ xuống) trong khu vực được giới hạn bởi vĩ tuyến với vĩ độ $|\varphi|>35^{\circ} 16^{\prime}$, thì từ công thức (2) lưu ý công thức (1) suy ra công thức chuyển độ cao chuẩn $\left(H_{M}^{\gamma}\right)_{m}$ trong hệ triều trung bịnh về độ cao chuẩn $\left(H_{M}^{\gamma}\right)_{2}$ trong hệ triều 0 :

$$
\left(H_{M}^{\gamma}\right)_{2}=\left(H_{M}^{\gamma}\right)_{m}+\Delta \rho_{0}
$$

Trong các tài liệu $[10,11]$ không luận chứng cho cơ sở khoa học của việc sử dụng công thức (2) khi tính đến hiệu ứng triều của Quả đất. Trong bài báo khoa học này chúng ta sẽ nghiên cứu lý thuyết tác động của hiện tượng triều và kiểm tra sự đúng đắn của công thức (4) dùng để chuyển độ cao chuẩn từ hệ triều trung bình về hệ triều 0 . Đây là điều rất quan trọng làm cơ sở cho việc giải quyết các bài toán hoàn thiện hệ độ cao quốc gia và xây dựng mô hình Quasigeoid ở nước ta.

\section{Giải quyết vấn đề}

Việc nghiên cứu hiện tượng triều được bắt đầu từ năm 1927 bởi Love $A$. E. H [1] và chủ yếu đối với lĩnh vực hải dương học. Việc nghiên cứu ảnh hưởng của hiện tượng triều đến các kết quả đo đạc được quan tâm mạnh mẽ vào cuối thập kỷ 60 và đầu thập kỷ 70 của Thế kỷ $X X$, ví dụ [3]. Tuy nhiên các công trình nghiên cứu về hiện tượng triều ít được phản ánh trong các sách chuyên môn, quy phạm đo đạc. Do đó rất thiếu các mô tả tường minh vể ảnh hưởng của hiện tượng triều đến việc thay đổi mặt đẳng thế và mặt vật lý của Quả đất. Điều này có thể dẫn đến việc đưa ra các công thức hiệu chỉnh các kết quả đo đạc do ảnh hưởng của hiện tượng triều khác nhau. Việc mô tả tương đối hợp lý tác động của hiện tượng triều đến sự biến thiên của mặt đẳng thế và mặt vật lý của Quả đất được cho trong tài liệu [5] và được trình bày ở hình 1 dưới đây.

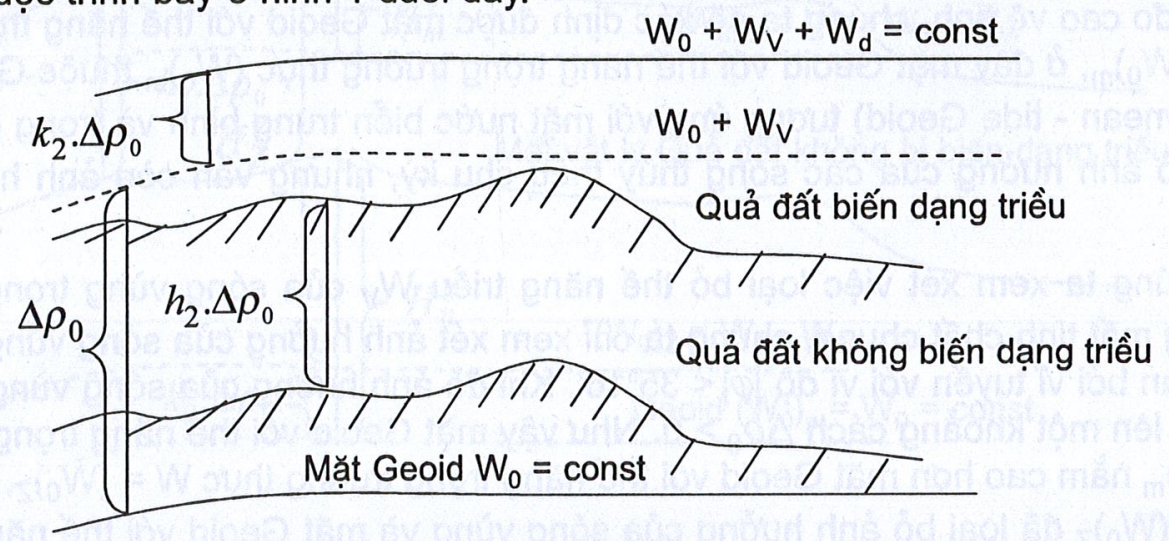

Hinh 1: Độ nâng của mặt đẳng thế W và mặt vật lý Quả đất dưới tác động của triều Quả đất 
Tác động của hiện tượng triều được giải thích như sau [5]: Dưới hiện tượng triều Quả đất mặt đẳng thế $W$ tương ứng với Quả đất không biến dạng triêu sẽ bị nâng lên 1 đoạn $\Delta \rho_{0}$ đến mức mặt đẳng thế $W+W_{V}$, còn bản thân bề mặt vật lý của Quả đất được nâng lên 1 đoạn $h_{2} \cdot \Delta \rho_{0}$ ở đây $h_{2}$ là số Love thứ nhất và theo tài liệu [2] $h_{2} \cong 0,62$. Việc nâng lên của mặt vật lý Quả đất được hiểu là hiệu ứng triều gián tiếp do sự đàn hồi của bề mặt Quả đất. Về phần mình nó sẽ dẫn đến sự phân bố lại vật chất trên lục địa và các đại dương và gây ra thêm thế biến dạng (deformation Potential) $W_{\mathrm{d}}$. Thế này lại làm mặt đẳng thế xê dịch đi một đoạn $k_{2} \cdot \Delta \rho_{0}$ đến mức thế $W+W_{V}+W_{d}$, ở đây $k_{2}$ là số Love thứ hai và theo tài liệu [2] $k_{2}$ $\cong 0,29$.

Việc mô tả trong tài liệu [5] hoàn toàn làm rõ tác động của hiện tượng triều đến trạng thái của mặt Geoid và mặt vật lý của Quả đất, nhưng chưa làm rõ trạng thái của các mặt nêu trên trong các hệ triều khác nhau. Điều này sẽ gây ra những quan niệm khác nhau khi giải quyết bài toán chuyển độ cao chuẩn giữa các hệ triều.

Chúng ta sẽ nghiên cứu giải quyếi vấn đề nêu trên ở phần tiếp sau đây: Mặt các biển và các đại dương thế giới chiếm $3 / 4$ diện tích Quả đất chịu ảnh hưởng mạnh của hiện tượng triều dưới sức hút của Mặt trăng và Mặt trời cùng với việc xuất hiện các loại sóng thủy triều. Mặt Geoid được chọn làm mặt khởi tính cho Hệ độ cao quốc gia liên quan chặt chẽ với mặt nước trung bình trên các biển và các đại dương thế giới và được xác định nhờ phương pháp đo cao vệ tinh (altimetry). Mặt Geoid là inặt gần sát với mặt nước trung bình trên các biển và các đại dương thế giới với độ chênh tư $1-2 m$ tùy theo các khu vực khác nhau trên đại dương thế giới. Do sự xuất hiện các sóng thủy triều với các biên độ thay đổi theo thời gian khác nhau trong một ngày đêm dưới sức hút của Mặt trăng và Mặt trời, nên thế năng thực W0 của mặt Geoid cũng biến đổi theo. Vì ciộ cao Geoid và độ cao chuẩn được xác định từ mặt Geoid, nên các giá trị của chúng tất yêu sẽ sẽ bị thay đổi dưới tác động của hiệu ứng triều trực tiếp dưới sức hút của Mặt trăng và Mặt trời.

Khi xác định mặt nước biển trung bình từ kết quả đo mực nước trên trạm nghiệm triều hoặc xác định mặt nước biển trung bình và mặt Geoid từ kết quả đo cao vệ tinh (altimetry) với khoảng thời gian đo liên tục lớn hơn 18,5 năm, mặt nước biển trung bình xác định được đã loại bỏ ảnh hưởng của các loại sóng thủy triều thay đổi theo các chu kỳ (hiệu ứng triều trực tiếp chu kỳ), nhưng ảnh hưởng của sóng vùng (hiệu ứng triều trực tiếp thường trực) vẫn chưa bị loại bỏ. Do đó khi xác định mặt nước biển trung bình và mặt Geoid nhờ phương pháp đo cao vệ tinh, chúng ta sẽ xác định được mặt Geoid với thế năng trọng trường thực $W=\left(W_{0}\right)_{m}$, ở đây mặt Geoid với thế năng trọng trường thực $\left(W_{0}\right)_{m}$ thuộc Geoid triều trung bình (mean - tide Geoid) tương ứng với mặt nước biển trung bình và trong giá trị $\left(W_{0}\right)_{m}$ đã loại bỏ ảnh hưởng của các sóng thủy triểu chu kỳ, nhưng vẫn còn ảnh hưởng của sóng vùng.

Chúng ta xem xét việc loại bỏ thế năng triều $W_{V}$ của sóng vùng trong giá trị $\left(W_{0}\right)_{m}$. Không mất tính chất chung, chúng ta chỉ xem xét ảnh hưởng của sóng vùng trong khu vực giới hạn bởi vĩ tuyến với vĩ độ $|\varphi|<35^{\circ} 16^{\prime}$. Khi đó ảnh hưởng của sóng vùng làm nâng mặt Geoid lên một khoảng cách $\Delta \rho_{0}>0$. Như vậy mặt Geoid với thế năng trọng trường thực $W$ $=\left(W_{0}\right)_{m}$ nằm cao hơn mặt Geoid với thế năng trọng trường thực $W=\left(W_{0}\right)_{Z}$, ở đây trong đại lượng $\left(W_{0}\right)_{z}$ đã loại bỏ ảnh hưởng của sóng vùng và mặt Geoid với thế năng trọng trường thực $W=\left(W_{0}\right)_{z}$ thuộc Geoid triều 0 (Zero - tide Geoid). Điều này có nghĩa là giá trị của thế 


\section{Mghiên cuím - Cíng dunng}

năng $\left(W_{0}\right)_{z}$ lớn hơn giá trị của thế năng $\left(W_{0}\right)_{m}$. Lưu ý thế năng triều $W_{V}$ trong công thức $(3)$ luôn dương đối với khu vực giới hạn bởi vĩ tuyến với vĩ độ $|\varphi|<35^{\circ} 16^{\prime}$, chúng ta suy ra quan hệ giữa các đại lượng $\left(\mathrm{W}_{0}\right)_{\mathrm{m}}$ và $\left(\mathrm{W}_{0}\right)_{z}$

$$
\left(W_{0}\right)_{m}=\left(W_{0}\right)_{z}-W_{V}
$$

Do sự đàn hồi của bề mặt Quả đất, nên dưới tác động của sóng vùng mặt vật lý của Quả đất được nâng lên 1 đoạn $h_{2} \cdot \Delta \rho_{0}$. Điều này về phần mình gây ra phân bố lại vất chất trong lòng Quả đất và cũng làm thay đổi thế năng của mặt Geoid đi một đại lượng bằng thế biến dạng $W_{d}$ và gây ra hiệu ứng triều gián tiếp. Do đó thực tế các công tác đo đạc và tính toán trắc địa đều liên quan đến mặt Geoid bi ảnh hưởng của hiệu ứng triều gián tiếp với thế năng $W_{0}-W_{d}$ trong hệ triều 0 . Khi lưu ý công thức (5), chúng ta thấy rằng mặt Geoid với thế năng trọng trường thực $\left(W_{0}\right)_{z}=W_{0}-W_{d}$ thuộc Geoid triều 0 và với mặt Geoid với thế năng trọng trường thực $\left(W_{0}\right)_{m}=W_{0}-W_{d}-W_{V}$ thuộc Geoid triều trung bình. Nếu loại bỏ ảnh hưởng của thế năng biến dạng $W_{d}$ trong thế năng $\left(W_{0}\right)_{z}$, thì chúng ta sẽ có mặt Geoid với thế năng trọng trường thực $W=\left(W_{0}\right)_{n}=W_{0}$ thuộc Geoid không phụ thuộc triều.

Dưới tác động của hiệu ứng triều gián tiếp, mặt Geoid với thế năng trọng trường thực $\left(W_{0}\right)_{n}=W_{0}$ trong hệ không phụ thuộc thủy triều sẽ bị nâng cao lên một đoạn $k_{2} \cdot \Delta \rho_{0}$ đến mức thế năng trọng trường thực $\left(W_{0}\right)_{Z}=W_{0}-W_{d}$ của mặt Geoid trong hệ triều 0 . Dưới tác động của sóng vùng (hiệu ứng triều trực tiếp thường trực), mặt Geoid với thế năng trọng trường thực $\left(W_{0}\right)_{z}$ trong hệ triều 0 sẽ bị nâng cao lên một đoạn $\Delta \rho_{0}>0$ đến mức thế năng trọng trường thực $\left(W_{0}\right)_{m}=\left(W_{0}\right)_{z}-W_{V}$ của mặt Geoid trong hệ triều trung bình. Với cách giải thích nêu trên chúng ta có hình 2 ở dưới đây.

Do khi xem xét các đại lượng cần quan tâm (độ cao trắc địa, độ cao chuẩn) trong hệ triều 0 , chúng ta vẫn có quan hệ với điểm $\mathrm{M}$ nằm trên mặt vật lý Quả đất bị biến dạng triều. Do đó thế năng thực tại điểm $M$ trên mặt vật lý Quả đất bị biến dạng triều trong hệ triều trung bình và hệ triều 0 là như nhau, tức

$$
\left(W_{M}\right)_{m}=\left(W_{M}\right)_{Z}
$$

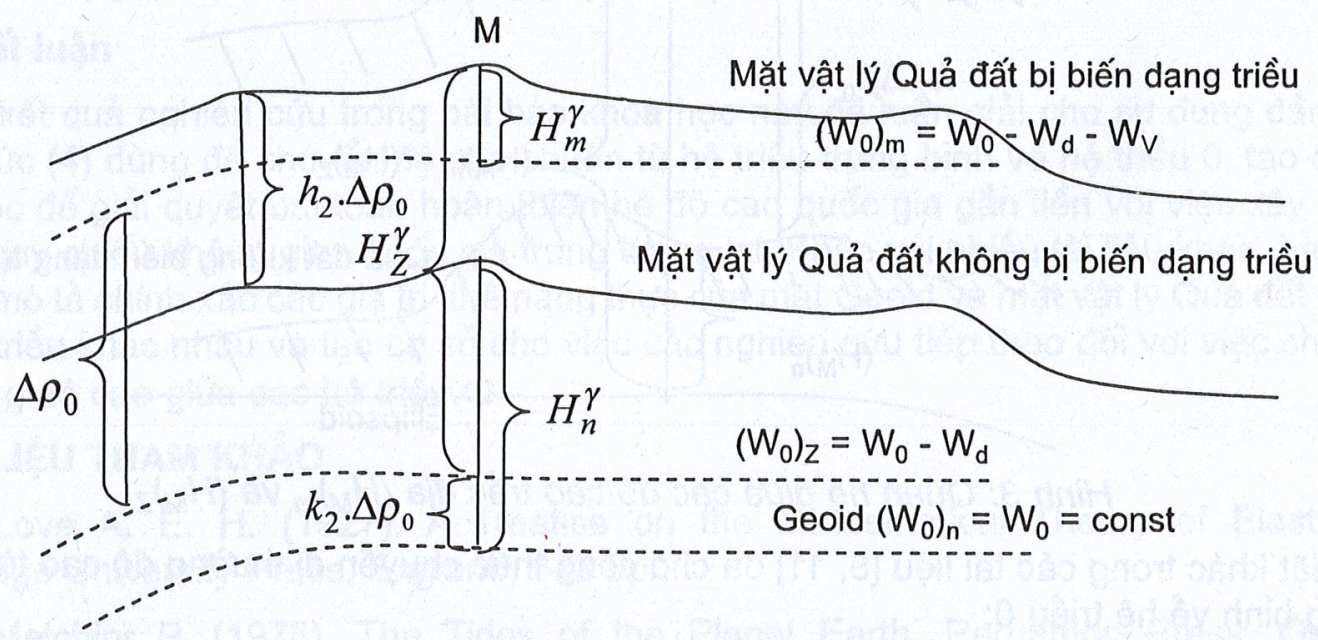

Hinh 2: Quan hệ giữa các độ cao chuẩn trong các hệ triều 


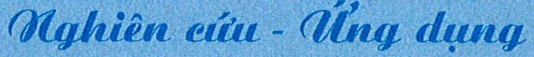

Từ hình 2 chúng ta có thể xác định được công thức chuyển độ cao chuẩn từ hệ triều trung bình về hệ không phụ thuộc như sau:

$$
\left(H_{M}^{\gamma}\right)_{n}=\left(H_{M}^{\gamma}\right)_{m}+\left(1+k_{2}-h_{2}\right) \Delta \rho_{0} .
$$

Công thức (7) hoàn toàn trùng với công thức được cho trong tài liệu [5]. Tiếp theo từ hình 2 chúng ta xác định công thức chuyển độ cao chuẩn từ hệ triều trung bình về hệ triều 0 :

$$
\left(H_{M}^{\gamma}\right)_{Z}=\left(H_{M}^{\gamma}\right)_{m}+\Delta \rho_{0}
$$

Chúng ta thấy rằng công thức (8) hoàn toàn trùng với công thức (4). Chúng ta sẽ chứng minh công thức (8). Trên cơ sở các công thức (5), (6) chúng ta sẽ thiết lập mối quan hệ giữa độ cao chuẩn $\left(H_{M}^{\gamma}\right)_{m}$ của điểm $\mathrm{M}$ trong hệ triều trung bình và độ cao chuẩn $\left(H_{M}^{\gamma}\right)_{Z}$ của điểm đó trong hệ triêuu 0

$$
\begin{aligned}
& \left(H_{M}^{\gamma}\right)_{Z}=\frac{\left(W_{0}\right)_{Z}-\left(W_{M}\right)_{Z}}{\bar{\gamma}}=\frac{\left(W_{0}\right)_{m}-\left(W_{M}\right)_{m}}{\bar{\gamma}}+\frac{W_{V}}{\bar{\gamma}}= \\
& =\left(H_{M}^{\gamma}\right)_{m}+\Delta \rho_{0} .
\end{aligned}
$$

Công thức (9) hoàn toàn trùng với công thức (8). Chúng ta chứng minh công thức (8) theo cách khác. Độ cao trắc địa $\mathrm{HM}$ của điểm $\mathrm{M}$ trên mặt vật lý của Quả đất là khoảng cách theo đường vuông góc giữa điểm đó và mặt Ellipsoid. Dưới tác động của sóng vùng, mặt vật lý Quả đất được nâng lên một đoạn $h_{2} \cdot \Delta \rho_{0}$ nhưng mặt Ellipsoid không bị dịch chuyển bởi tác động của sóng vùng (xem mô tả trên hình 3 dưới đây). Mặt khác khi xác định độ cao trắc địa trong hệ triều trung bình và hệ triều 0 chúng ta vẫn liên quan đến điểm $M$ nằm trên mặt vật lý Quả đất bị biến dạng triều. Do đó trong các hệ triều trung bình và hệ triều 0 độ cao trắc địa không thay đổi, tức

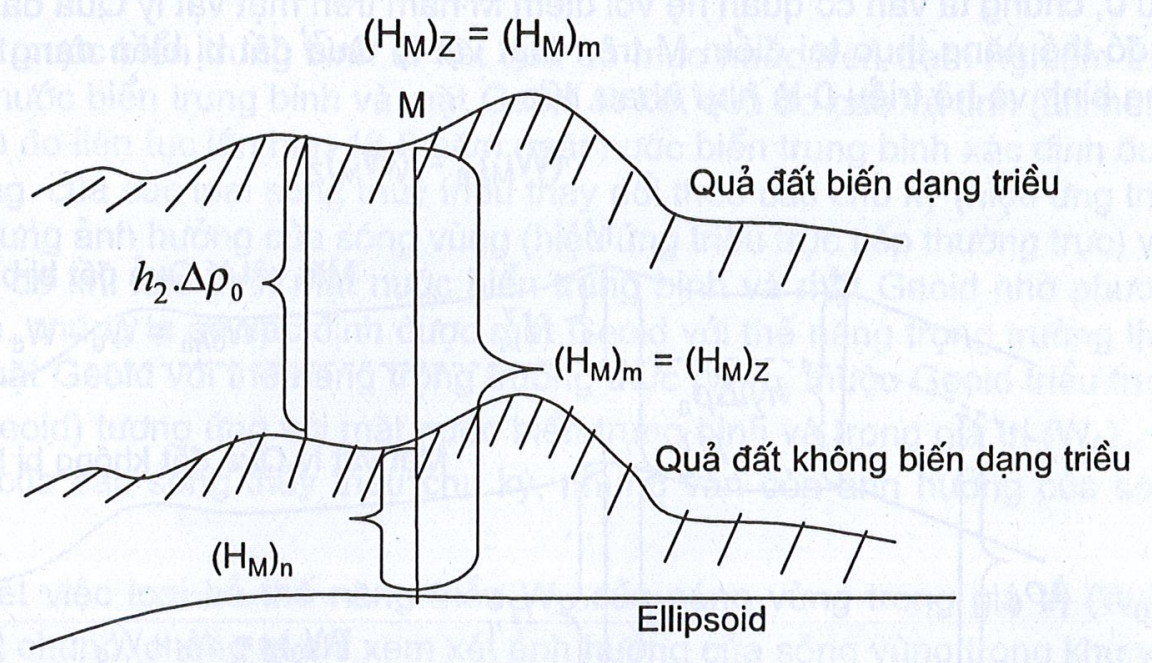

Hình 3: Quan hệ giữa các độ cao trắc địa $\left(H_{M}\right)_{m}$ và $\left(H_{M}\right)$

Mặt khác trong các tài liệu $[8,11]$ đã cho công thức chuyển dị thường độ cao từ hệ triều trung bình về hệ triều 0 :

$$
\left(\zeta_{M}\right)_{Z}=\left(\zeta_{M}\right)_{m}-\Delta \rho_{0}
$$


Từ các công thức (10) và (11) chúng ta có

$$
\begin{aligned}
& \left(H_{M}^{\gamma}\right)_{Z}=\left(H_{M}\right)_{Z}-\left(\zeta_{M}\right)_{Z}=\left(H_{M}\right)_{m}-\left(\zeta_{M}\right)_{m}+\Delta \rho_{0}= \\
& =\left(H_{M}^{\gamma}\right)_{m}+\Delta \rho_{0} .
\end{aligned}
$$

Công thức (12) hoàn toàn trùng với công thức (8). Như vậy bằng các cách chứng minh khác nhau, chúng ta đi đến kết luận rằng công thức (8) là công thức đúng đắn dùng để chuyển độ cao chuẩn từ hệ triều trung bình về hệ triều 0 .

Trong trường hợp bình sai mạng lưới thủy chuẩn quốc gia trong hệ triều 0 , chênh cao đo $\mathrm{h}_{\mathrm{ij}}$ giữa hai mốc thủy chuẩn $\mathrm{i}$ và $\mathrm{j}$ được chuyển từ hệ triều trung bình về hệ triều 0 theo công thức:

ở đây

$$
\left(h_{i j}\right)_{Z}=\left(h_{i j}\right)_{m}+\delta h_{i J}, \quad<m>
$$

$$
\delta h_{i J}=-\frac{288410 .\left(\operatorname{Sin}^{2} B_{j}-\operatorname{Sin}^{2} B_{i}\right)+1950 . .\left(\operatorname{Sin}^{4} B_{j}-\operatorname{Sin}^{4} B_{i}\right)}{\bar{\gamma}}<m>,
$$

$\bar{\gamma}$ - giá trị trung bình của lực trọng chuẩn giữa hai mốc $\mathrm{i}$ và $\mathrm{j}$.

Từ hình vẽ 3 chúng ta suy ra được công thức chuyển độ cao trắc địa từ hệ không phụ thuộc triều (non - tide system) về hệ triều 0 như sau

$$
\left(H_{M}\right)_{Z}=\left(H_{M}\right)_{n}+h_{2} \cdot \Delta \rho_{0} \text {. }
$$

Trong thực tế hiện nay, khi sử dụng các phần mềm hiện đại, ví dụ phần mềm Bernese để xử lý các dữ liệu đo GNSS (Global Navigation Satellite System) trong ITRF chúng ta đã hiệu chỉnh vị trí của máy thu GNSS vào mỗi thời điểm thu tín hiệu vệ tỉnh do ảnh hưởng của hiện tượng triều Quả đất. Do đó các kết quả xử lý mạng lưới GNSS trong ITRF đã cho độ cao trắc địa của các điểm GNSS trong hệ không phụ thuộc triều. Trong trường hợp này để xác định dị thường độ cao GNSS/thủy chuẩn trong hệ triều 0 phải sử dụng công thức (13) để chuyển độ cao trắc địa từ hệ không phụ thuộc triều (non - tide system) về hệ triều 0.

\section{Kết luận}

Các kết quả nghiên cứu trong bài báo khoa học này đã luận giải cho sự đúng đắn của công thức (4) dùng để chuyển độ cao chuẩn từ hệ triều trung bình về hệ triều 0 , tạo cơ sở khoa học để giải quyết bài toán hoàn thiện hệ độ cao quốc gia gắn liền với việc xây dựng Khung quy chiếu không gian quốc gia trong tương lai. Khác với nhiều tài liệu khác, bài báo này đã mô tả chính xác các giá trị thế năng thực của mặt Geoid và mặt vật lý Quả đất trong các hệ triều khác nhau và tạo cơ sở cho việc các nghiển cứu tiếp theo đối với việc chuyển dị thường độ cao giữa các hệ triều. $O$

\section{TÀI LIẸU THAM KHẢO}

[1]. Love A. E. H. (1927). A Treatise on the Mathematical Theory of Elasticuty. Cambridge University Press, England, 643 pp.

[2]. Melchior P. (1978). The Tides of the Planet Earth. Pergamon Press, Oxford, England. $620 \mathrm{pp}$. 
[3]. Harrison J.C. (1978). Earth Tides and Geodesy. Procceedings of the GEOP-9 Conference, Columbus, Ohio.

[4]. luzephovich A. P., Ogorodova L.V. (1980). Trọng lực. Matxcơva, Nedra, 320 trg. (Tiếng Nga).

[5]. Vani ček P. (1980). Tidal Corrections to Geodetic Quantities. NOAA Technical Report NOS 83 NGS 14, National Geodetic Survey, Rockvile, Md. U.S. Department of Commerce, National Oceanic and Atmospheric Administration, National Ocean Survey.

[6]. Baker T. F. Tidal Deformation of the Earth. Science Progress. Oxford, 69, PP. 197 233. 1984.

[7]. International Association of Geodesy (IAG). (1984). IAG Resolutions adopted at the XVIII General Assembly of the IUGG in Hamburg, August 1983. Bulletin Geodetique, 58(3), "The Geodesist's handbook", p. 321.

[8]. Ekman, M.. (1989). Impacts of Geodynamic Phenomena on Systems for Height and Gravity, Bulletin Géodésique, 63(3), 281-296, 1989.

[9]. Rapp, R.H., R.S. Nerem, C.K. Shum, S.M. Klosko, and R.G. Williamson, (1991). Consideration of Permanent Tidal Deformation in the Orbit Determination and Data Analysis for the Topex/Poseidon Mission, NASA TM 100775, Goddard Space Flight Center, Greenbelt, MD, 1991.

[10]. M $\ddot{a}$ kinen J.(2008). The treatment of the permanent tide in EUREF products. EUREF Symposium, June 17 - 21 2008, Brussels.

[11]. Sacher M., Ihde J., Liebsch G., Mäkinen J. (2008). EVRF2007 as Realization of the European Vertical Reference System. EUREF Symposium, June 17 - 21 2008, Brussels. O

\section{Summary}

PERFECTION OF A FORMULA FOR TRANSFORMATION OF THE NORMAL HEIGHT FROM MEAN TIDE SYSTEM TO ZERO - TIDE SYSTEM

Ass. Prof. Dr.Sc. Ha Minh Hoa

Eng. Nguyen Thi Thanh Huong

Vietnam Institute of Geodesy and Cartography

This scientific article realized accurate description of the Geopotentials of the Geoid and the Earth physical surface in the different tide systems and proved the formula for transformation of the normal height from mean tide system to zero - tide system. The Research results in this article created the base for solving task of a perfection of the state height system in connection with a building of state spatial reference frame in the future. $O$ 